\title{
Vegetación leñosa, endemismos y estado de conservación en los bosques estacionalmente secos de Jaén, Perú
}

\author{
Woody vegetation, endemism and conservation status in the seasonally \\ dry forests of Jaen, Peru
}

\author{
José Luis Marcelo-Peña
}

Herbario MOL. Facultad de Ciencias Forestales. Universidad Nacional Agraria La Molina. Lima Perú. E-mail:

jlmarcelop@lamolina.edu.pe

\begin{abstract}
Resumen
Son presentados los resultados de ocho inventarios botánicos rápidos realizados en los relictos del bosque tropical estacionalmente seco (BTES) de los alrededores de la ciudad de Jaén, Cajamarca, Perú; ubicados entre los $5^{\circ} 38^{\prime}$ y $5^{\circ} 48^{\prime}$ de latitud Sur y los $78^{\circ} 41^{\prime}$ y $78^{\circ} 48^{\prime}$ longitud Oeste, las alturas oscilan entre 615 y 1100 $\mathrm{m}$. El estudio reportó un total de 151 especies, 103 géneros y 40 familias de plantas leñosas. El área estudiada presenta una mayor diversidad en contraste con los BTES de regiones vecinas, en particular presenta el mayor número de endemismos en los BTES del Sur Occidente del Ecuador y Perú. Sin embargo, la zona está seriamente amenazada por el impacto antrópico que demanda especies leñosas para material combustible, áreas para cultivos agrícolas, ganadería extensiva y vivienda.
\end{abstract}

Palabras claves: Evaluaciones rápidas, bosque relictuales, Bosque Tropical Estacionalmente Seco, flora, endemismos, conservación.

\section{Abstract}

Eight relictual areas of seasonally dry tropical forest (SDTF) from the surroundings Jaen city, in Cajamarca, were investigated using a rapid inventory technique. The areas were situated between $5^{\circ} 38^{\prime}$ and $5^{\circ} 48^{\prime} \mathrm{S}$ and $78^{\circ} 41^{\prime}$ and $78^{\circ} 48^{\prime} \mathrm{W}$, and between 615 and $1100 \mathrm{~m}$ altitude. A total of 151 species, 103 genera and 40 families of woody plants were recorded. The area shows high diversity of woody plants in comparison with other neighbouring regions. Most notable is its endemism, which is the highest in the region of southwest Ecuador and Peru. However, the area is seriously threatened by demand for firewood and the need for land for crops, large cattle farms and housing.

Keywords: Rapid inventories, seasonally dry tropical forest, woody flora, endemism, conservation. $\begin{array}{ll}\text { Presentado: } & 20 / 07 / 2007 \\ \text { Aceptado: } & 15 / 04 / 2008\end{array}$ Publicado online: $\quad 21 / 07 / 2008$

\section{Introducción}

En este trabajo adoptamos la definición amplia de Bosques Tropicales Estacionalmente Secos (BTES), propuesta por Pennington et al. (2000). Los BTES son ecosistemas dominados por árboles, mayoritariamente caducifolios durante la estación seca, consecuentemente los procesos ecológicos son moderadamente estacionales y la productividad primaria neta es menor que en los bosques húmedos, por presentarse solo en la temporada de lluvias. Estos bosques son además de menor altura y área basal que los bosques tropicales húmedos, se presentan en suelos relativamente fértiles donde la precipitación es menor de $1600 \mathrm{~mm}$ anuales y donde hay una estación seca fuertemente definida de al menos 5 a 6 meses del ańo, periodo en el que reciben menos de $100 \mathrm{~mm}$ (Gentry, 1995). Esta característica muestra un marcado contraste con los bosques tropicales lluviosos que presentan regímenes climáticos más húmedos y con más de $100 \mathrm{~mm}$ de precipitación en todos los meses del año (Whitmore, 1998).

Según el Mapa Forestal del Perú (INRENA, 1995), el bosque seco abarca un área de $39,451 \mathrm{~km}^{2}$ (3,07\% de la superficie del territorio peruano), con mayor extensión en los departamentos de Lambayeque, Piura y Tumbes, pero, existen fragmentos y remanentes de bosque estacionalmente seco a lo largo de todo el Perú. Los BTES han recibido poca atención por parte de conservacionistas y ecólogos comparados con los bosques lluviosos, a pesar de que los BTES son los más amenazados. La razón principal de la destrucción masiva de este ecosistema es la naturaleza fértil de su suelo, el cual es apreciado para la agricultura (Ratter et al., 1978). Su destrucción es exacerbada por las poblaciones humanas presentes en muchas zonas de vida de bosque seco neotropical (Murphy \& Lugo, 1995).

Bridgewater et al. (1999), mencionan que durante los últimos años los bosques secos del Norte del Perú en Cajamarca y Amazonas, notablemente en el valle del Marañón, han sido ocupados progresivamente por cientos de familias, quienes al encontrar valles fértiles los han intervenido y destruido para desarrollar actividades agrícolas y ganaderas. Por ejemplo, en la provincia de Jaén, el valle en donde se asienta la ciudad del mismo nombre estaba completamente cubierto de vegetación estacionalmente seca. Sin embargo, con el transcurrir de los años se ha producido una alta demanda por áreas fértiles para instalar cultivos de arroz, cacao y frutales, así como, de un número considerable de especies leñosas utilizadas como material combustible y otras para la construcción de viviendas, que han propiciado la destrucción de la vegetación, quedando bosques relictuales que en la actualidad se encuentran seriamente amenazados y que necesitan urgentemente ser documentados.

Estudios de los BTES del Norte del Perú han sido realizados por Weberbauer (1945); Ríos (1989); Ferreyra (1996); Gentry (1995); Bridgewater et al., (2003), Linares-Palomino (2004) y Marcelo-Peña et al. (2007). Sin embargo, son escasos los estudios de la flora leñosa del BTES del distrito de Jaén.

En el presente estudio realizo un análisis de la composición florística de los BTES del distrito de Jaén, se identifican las localidades que presentan altos niveles de endemismos y se describe los riesgos potenciales de extinción por la influencia antrópica. Así mismo, se espera que los resultados sean un marco de referencia importante para entender y contextualizar globalmente los diferentes niveles de diversidad y endemismos de los BTES, además de propiciar las bases para la conservación de los Bosques Tropicales Estacionalmente Secos.

\section{Descripción de la zona de estudio}

La provincia de Jaén es una de las 13 provincias que conforman la región Cajamarca, se encuentra ubicada al Norte de esta región, pose una superficie de $5232 \mathrm{~km}^{2}$. Su capital, la ciudad de 

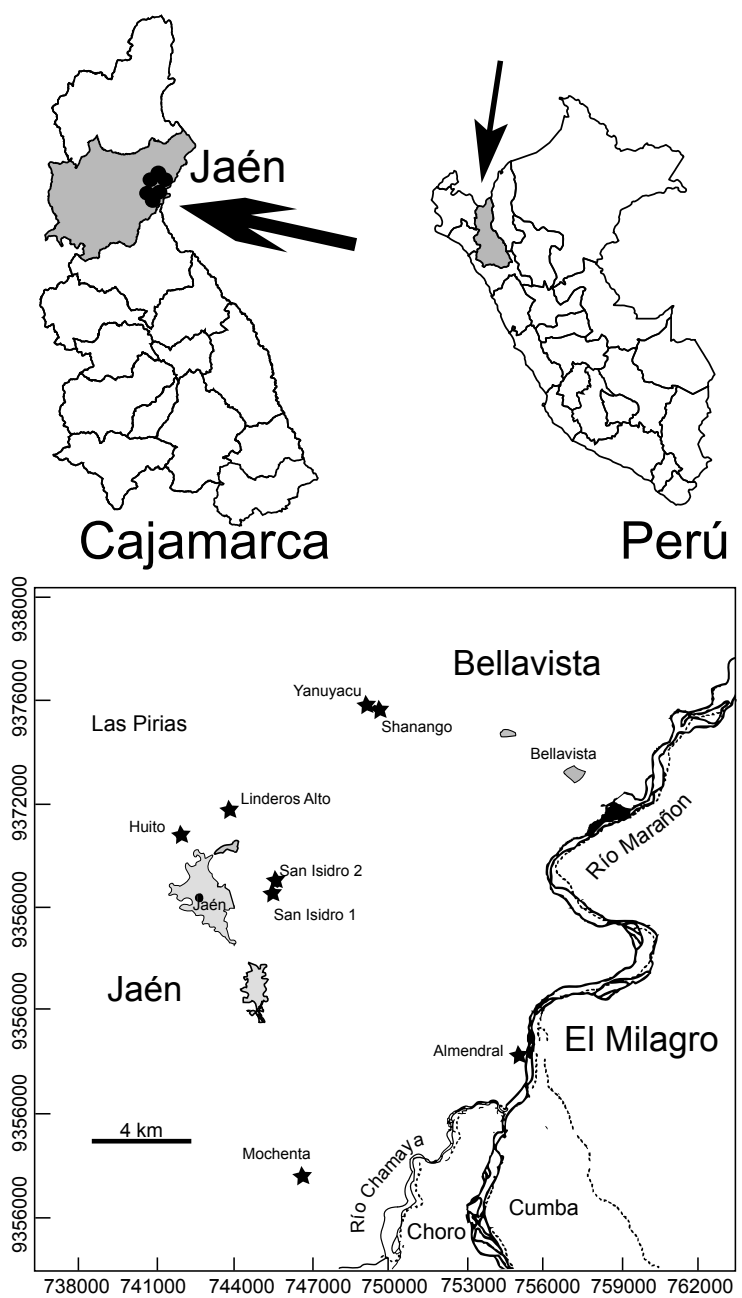

Figura 1. Ubicación del área de estudio. Las estrellas señalan los lugares muestreados.

Jaén se encuentra entre las coordenadas $05^{\circ} 42^{\prime} S$ y $78^{\circ} 48^{\prime} \mathrm{W}$. La zona estudiada se ubico entre los 615 y $1100 \mathrm{~m}$ de altitud y los 5³8' y 548' S y los 7841' y 7848' W; incluyo los lugares El Huito, San Isidro 1, San Isidro 2, Yanuyacu, Shanango, Linderos Alto, El Almendral y Mochenta (Figura 1).

La zona de estudio se encuentra dentro de la zona de vida Bosque muy seco tropical (bms- T), esta ocupada por especies perennifolias y caducifolias de porte arbóreo y arbustivo. El clima es seco con una temperatura media anual que oscila entre 24 a $26{ }^{\circ} \mathrm{C}$, con máximas que alcanzan los $30^{\circ} \mathrm{C}$ y mínimas que están alrededor de 20 a $21^{\circ} \mathrm{C}$, la temperatura promedio se mantiene a lo largo del año. La precipitación promedio anual varía desde 350 hasta $1000 \mathrm{~mm}$ y presenta un periodo seco entre los meses de mayo a octubre y de mayores precipitaciones entre octubre y abril (Ediciones Peisa, 2004).

El escenario fisiogeográfico presenta rasgos geomorfológicos que son el resultado de la evolución originada por factores tectónicos y erosiónales. Está conformada por laderas moderadamente empinadas de $25-50 \%$ de pendiente y laderas muy empinadas mayores de 70\%. La zona de estudio ocupa el ámbito de la cuenca Amojú que se encuentra disectada por el cauce del río del mismo nombre, fuente primaria proveedora de agua para riego y para satisfacer las necesidades de las poblaciones asentadas en el valle de Jaén. El recorrido de las aguas del río Amojú son de Norte a Sur y aguas abajo confluyen en la margen izquierda del río Marañón que es el colector principal.
El valle de Jaén es eminentemente agrícola y en gran parte de su área se ha instalado cultivos de "arroz" Oriza sativa L. Adicionalmente se cultiva "cacao" Theobroma cacao L. y frutales en menores áreas. Los cultivos de "maíz" Zea maiz Vell., "plátano" Musa spp. y "yuca” Manihot esculenta Cif., son principalmente de consumo familiar y/o para el mercado local. Las zonas de ladera presentan agricultura restringida dedicada especialmente al cultivo de "maíz" y a la siembra de pasto para sostener ganadería extensiva. Todos los relictos evaluados están siendo utilizados actualmente como áreas de pastoreo de ganado vacuno. Por ejemplo, alrededor del relicto ubicado al Noroeste de la ciudad de Jaén, en el sector El Huito se han establecido pastos que regularmente son quemados y presentan una formación tipo sabana.

\section{Material y métodos}

Los inventarios botánicos rápidos (IBR) se desarrollaron a partir de abril y julio de 2006 (Huito, San Isidro1, Yanuyacu y Shanango), culminando las evaluaciones en diciembre de 2006, enero y abril de 2007 (El Almendral, Mochenta, Linderos Alto y San Isidro 2). En los IBR se siguió la metodología de Hawthorne \& Abu-Juam (1995) y Gordon et al. (2004) con modificaciones. Sólo se tomó el registro de las especies leñosas con un diámetro de tronco a nivel del suelo $\geq 2,5 \mathrm{~cm}$ (se incluyeron las trepadoras). El tiempo de duración de cada evaluación fue de 90 minutos. El equipo de trabajo estuvo compuesto por dos personas: un botánico y un ayudante de campo. Dentro del relicto elegido se caminó sin seguir una dirección establecida, la prioridad fue el registro de una nueva especie leñosa. Sin embargo, la visualización abarcó aproximadamente $10 \mathrm{~m}$ (5 m a cada lado del recorrido) en áreas abiertas y con poca vegetación arbórea y de cuatro metros (dos metros a cada lado del recorrido) donde la vegetación fue más abundante, tupida y enmarañada; estimo que las áreas evaluadas oscilan entre una y cuatro hectáreas. Durante los primeros inventarios se colectaron todas las especies leñosas encontradas (estériles o fértiles), siguiendo la metodología descrita por Rodríguez \& Rojas (2002). Posteriormente sólo se colectó cuando se tuvo dudas de la identidad de la especie o si fueron nuevos registros. También se realizaron colectas de las especies leñosas que se encontraron en los recorridos hacia las zonas de evaluación. Para la determinación de las especies endémicas y datos de distribución se tomó como referencia las colecciones de los herbarios USM y MOL, también se revisó El Libro rojo de las plantas endémicas del Perú (León et al., 2006), el Catálogo de las Angiospermas y Gimnospermas del Perú (Brako \& Zarucchi, 1993), el Libro Rojo de las Plantas endémicas del Ecuador 2000 (Valencia et al., 2000), además de la revisión de monografías de listas confiables (Pendry, 2003; Barneby \& Grimes, 1997), y de bases de datos en línea W3TROPICOS (http://mobot.mobot. org/W3T/Search/vast.html).

Las colecciones botánicas fueron depositas en el Herbario MOL.

\section{Resultados}

Se realizaron un total de 413 colecciones botánicas que representan 151 taxones leńosos; 136 fueron identificados hasta especie $(88,7 \%), 16$ hasta género, uno hasta familia y uno es desconocido. Las listas completas de las especies registradas en los IBR y durante los recorridos a las aéreas de evaluación se detallan en el Apéndice 1. 


\section{Composición florística y diversidad}

Los resultados revelan un total de 151 especies, 103 géneros y 40 familias para los BTES del distrito de Jaén. La riqueza de especies de acuerdo a los hábitos de crecimiento muestra que 78 son arbustos, 56 árboles, 17 escandentes-lianescentes.

Las cinco familias más diversas son Leguminosae con 20 géneros y 36 especies, le siguen en orden decreciente: Cactaceae con 11 géneros y 15 especies, Euphorbiaceae con nueve géneros y 15 especies, Asteraceae con cinco géneros y cinco especies y Bignoniaceae con cuatro géneros y cinco especies. Las demás familias están representadas por menos de tres géneros y siete especies. Cabe anotar que la riqueza de especies se basa en los conocimientos que disponemos hasta el momento. No obstante, el número se incrementará cuando se amplíen los estudios a otras áreas del BTES del distrito de Jaén, y otros distritos de la provincia de Jaén que presentan BTES (Huabal, Las Pirias, Colasay, Sallique, Pomahuaca, Pucará, San Felipe, Chontalí, San José del Alto, Santa Rosa y Bellavista).

La familia mas diversa fue Leguminosae con el 23\% de las especies, le siguieron Cactaceae y Euphorbiaceae con el 10\%, Bignoniaceae con 5\%, Boraginaceae y Sapindaceae con $4 \%$ Asteraceae y Bombaceae 3\%. Los géneros más diversos fueron: Senna con seis especies; Acacia y Cordia con cinco especies; Bauhinia, Capparis, Croton, Espostoa y Serjania con cuatro especies. La distribución de las especies de estos géneros ocupa diferentes hábitats desde los más secos hasta los más "húmedos" (áreas donde hay mayor acumulación de agua por escorrentía o en lugares accesibles al agua subterránea).

Según los IBR la zona más diversa es Mochenta con 50 especies, le siguen en orden decreciente El Huito (47), San Isidro2 (46), San Isidro1 (42), Linderos Alto (40), Yanuyacu (39), Shanango (35) y El Almendral (29). (Fig. 2)

El sector Mochenta es el más diverso, debido a que abarcó mayor área, con aproximadamente $1000 \mathrm{~m}$ lineales de recorrido y cuatro metros de visualización (dos a cada lado) y cruzó diferentes escenarios fisiogeográficos: colina baja muy seca con vegetación predominantemente arbustiva, con especies de C. thurifer, Bougainvillea peruviana H. \& B., Vallesia glabra (Cav.) Link, Phitecellobium excelsum (Kunth) Benth., Tetrasida chachapoyensis (Baker f.) Fryxell \& Fuertes, Parkinsonia praecox

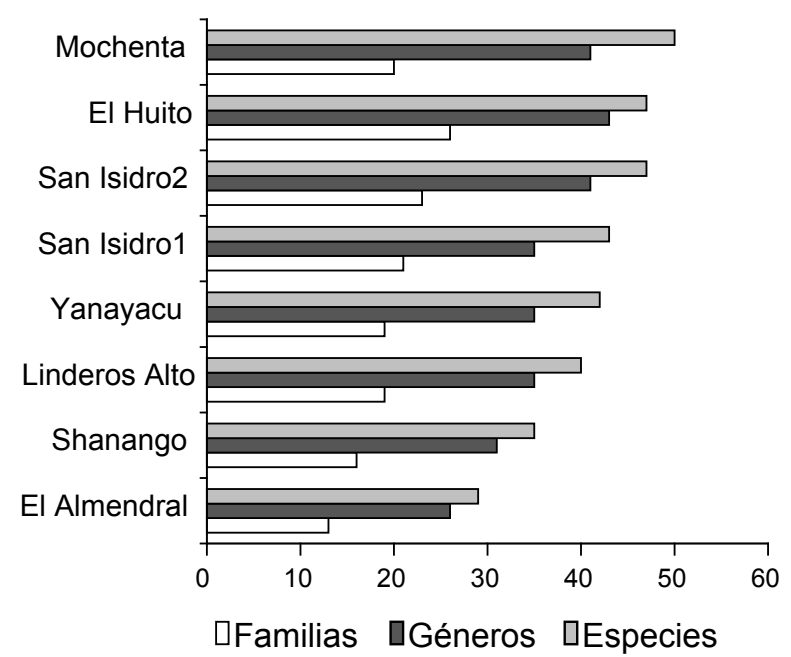

Figura 2. Número de familias, géneros y especies en los Bosques Tropicales Estacionalmente Secos evaluados, en Jaén, Perú.
(R. \& P.) Harms, Cyathostegia mattewsii (Benth.) Schery y Maytenus octogona (L' Her.) DC.; cabecera de colina baja muy seca con mayor densidad de cactáceas siendo las más conspicuas Browningia altissima (F. Ritter) Buxb., E. lanata, Praecereus euchlorus spp jaenensis (Rauh \& Backeb.) Ostolaza, Opuntia macbridei Britton \& Rose y Melocactus bellavistensis Rauh \& Backeberg; colina bajas con predominio de especies arbustivas y árboles dispersos de $A$. macracantha, Jacquinia mucronata Roem. \& Schult., C. flexuosa, y Eriotheca discolor (Kunth) A. Robyns, también terrazas onduladas y quebradas secas por donde drenan las aguas de las colinas, presentado una vegetación predominantemente arbórea dominada por Anadenanthera colubrina (Vell.) Brenan, B. altissima, C. flexuosa, Tabebuia chrysantha subsp meridionalis A. H. Gentry y Ceiba insignis (Kunth) P.E. Gibbs \& Semir.

El Huito concentra seis familias con dos géneros más que el sector Mochenta, su mayor rango altitudinal $\left(780 \_1100 \mathrm{~m}\right)$ propicia mejores condiciones para albergar géneros de amplio rango de distribución altitudinal como Celtis, Annona, Clusia y Maclura. Este sector presenta una formación más homogénea y se encuentra en colinas con pendientes moderadamente empinadas de $25-50 \%$ y laderas muy empinadas con pendientes mayores de $70 \%$; la vegetación es predominantemente arbórea con dominancia de $C$. iguaguana, $A$. macracantha, $A$. colubrina y Aspidosperma polyneuron Müll. Arg., en el estrato arbustivo se observa frecuentemente Urera caracasana (Jacq.) Griseb., Ruprechtia aperta Pendry, C. mattewsii, Rauhocereus riosaniensis Backeberg, P. euchlorus spp. jaenensis y una alta densidad de brinzales y latizales de $C$. iguaguana.

El Almendral es el sector menos diverso, presenta un paisaje dominado por cactáceas de porte arbóreo como Armatocereus rahuii Backeberg, $B$. altissima y E. lanata, árboles dispersos de $A$. macracantha, J. mucronata y E. discolor, en el estrato arbustivo se observa frecuentemente y Espostoa blossfeldiorum (Werdermann) Buxbaum, C. thurifer, M. pectinatipinna, Sideroxylon obtusifolium (Roem. \& Schult.) T.D. Penn., P. euchlorus spp. jaenensis y O. macbridei Britton \& Rose, que en algunas áreas es abundante y la zona se torna casi impenetrable por su presencia. Este sector es el más seco debido a las bajas precipitaciones por el encajonamiento del valle y por que las cadenas de montańas no permiten el paso de las nubes y las precipitaciones abundantes.

En general en los BTES del distrito de Jaén en zonas con evidencia de alteraciones antropogénicas frecuentes, la vegetación es de tipo matorral con alturas de dos a cuatro metros en donde las formas arbustivas son dominantes y se observa con frecuencia C. thurifer, C. matthewsii, T. chachapoyensis, Phitecellobium excelsum (Kunth) Benth. Acacia weberbaueri Harms, Mimosa pectinatipinna, $R$. riosaniensis y P. euchlorus spp. jaenensi entre los más conspicuas; asociados con árboles dispersos de C. flexuosa, C. iguaguana, Cordia saccellia Gottschling \& J.S. Mill., J. mucrona, S. obtusofolium, A. macracantha, C. insignis, B. altissima y, E. lanata; en las áreas más "húmedas" la formación es predominantemente arbórea pero existe una mixtura de especies de Leucaena trichodes (Jacq.) Benth., C. insignis, Albizia multiflora (Kunth) Barneby \& J.W. Grimes, A. colubrina, Triplaris cumingiana Fisch. \& C.A. Mey. ex C.A. Mey., Celtis loxensis C. C. Berg, Zanthoxylum rigidum Humb. \& Bonpl. ex Willd., Hura crepitans L., A. polyneuron, Maclura tinctorea (L.) Steud., Esenbeckia cornuta Eng., y otros. 


\section{Endemismos}

En los BTES la riqueza de especies es relativamente baja comparada con la encontrada en los bosques tropicales amazónicos. Sin embargo, se observa un alto grado de endemismo. Sagástegui et al. (1999), manifiestan que los niveles de endemismo en el Norte del Perú son extremadamente altos (incluyendo las regiones de Piura, Cajamarca, Amazonas y San Martín), con más de 715 especies y 11 géneros reconocidos como endémicos.

Un total de 32 especies endémicas fueron observadas en el presente estudio. El único género endémico reportado para la zona de estudio es Maraniona, especie observada con escasos individuos. Los sectores con mayor número de endemismos fueron San Isidro2, Mochenta y Shanango (12 spp. endemicas); le siguen en orden decreciente, San Isidro1 (11), Linderos Alto (10), El Huito y Yanuyacu (9) y El Almendral (8) (Tabla 1). Cabe anotar que en estos sectores, estas especies se pueden registrar en áreas no mayores de cinco hectáreas. Las familias más ricas en endemismos fueron Cactaceae (11 spp.), Leguminosae (7) y Euphorbiaceae (4).

\section{Discusión}

\section{De la diversidad y la composición florística}

Es difícil comparar la riqueza de especies con estudios florísticos realizados en otros territorios y con otras formaciones análogas. Las dificultades se deben a la escasa información y a la ausencia de criterios estandarizados en los métodos de evaluación, que garanticen una correcta confrontación.

Weberbaur (1945) y Ferreyra (1996), presentan listados florísticos breves de la vegetación de los bosques estacionalmente de la zona del Marañón. Ríos (1989) reporta: 179 especies de plantas vasculares, con 82 especies leńosas, del Coto de Caza El Angolo. Bridgewater et al. (2003), reportan un total de 110 especies para los BTES del Departamento de Amazonas, 89 especies para los BTES

Tabla 1. Especies endémicas registradas en los bosques tropicales estacionalmente secos (BTES) del distrito de Jaén, Cajamarca, Perú.Sectores Evaluados: A. El Huito, B. San Isidro 1, C. San Isidro 2, D. Shanango, E. Yanuyacu, F. El Almendral, G. Linderos Alto Y H. Mochenta.

\begin{tabular}{|c|c|c|c|c|c|c|c|c|c|c|c|}
\hline N. ${ }^{\circ}$ & Género y especie & Familia & A & B & $\mathrm{C}$ & $\mathbf{D}$ & E & $\mathbf{F}$ & G & $\mathbf{H}$ & Al. \\
\hline 1 & Monactis rhombifolia Sagast. \& Dillon & Asteraceae & $\mathrm{x}$ & $x$ & $x$ & $\mathrm{x}$ & & & $\mathrm{x}$ & & \\
\hline 2 & Onoseris cabrerae Ferreyra & Asteraceae & & & & & & & & & $x$ \\
\hline 3 & Cordia iguaguana Melch. ex I.M. Johnst. & Boraginaceae & $x$ & $x$ & $x$ & $x$ & $x$ & & $x$ & & \\
\hline 4 & Cordia varronifolia J. M. Johnson & Boraginaceae & & $\mathrm{x}$ & & & & & & $x$ & \\
\hline 5 & Armatocereus rahuii Backeb. & Cactaceae & & & & & & $x$ & & $x$ & \\
\hline 6 & Browningia altissima (F. Ritter) Buxb. & Cactaceae & $x$ & $x$ & $x$ & $x$ & $x$ & $x$ & $x$ & $x$ & \\
\hline 7 & Cleistocactus tenuiserpens Rauh \& Backeb. & Cactaceae & & & $x$ & & & & & & \\
\hline 8 & Espostoa blossfeldiorum (Werdermann) Buxbaum & Cactaceae & & & & $x$ & $x$ & $x$ & & & \\
\hline 9 & Espostoa mirabilis F. Ritter & Cactaceae & & & & & & & & $\mathrm{x}$ & \\
\hline 10 & Espostoa superba F. Ritter & Cactaceae & & & $\mathrm{x}$ & & & & & & \\
\hline 11 & Hylocereus microcladus Backeberg & Cactaceae & $\mathrm{x}$ & & & & & & & & \\
\hline 12 & Opuntia macbridei Britton \& Rose & Cactaceae & & $\mathrm{x}$ & $\mathrm{x}$ & & $\mathrm{x}$ & $\mathrm{x}$ & & & \\
\hline 13 & Praecereus euchlorus subsp. jaenensis (Rauh \& Backeb.) Ostolaza & Cactaceae & $x$ & $\mathrm{x}$ & $x$ & $x$ & $x$ & $x$ & $x$ & $x$ & \\
\hline 14 & Pereskia horrida (Kunth) DC. & Cactaceae & & & $x$ & & $\mathrm{x}$ & $x$ & $\mathrm{x}$ & $x$ & \\
\hline 15 & Pereskia horrida ssp. rauhii (Backeberg) Ostolaza & Cactaceae & & & & & & & & & $x$ \\
\hline 16 & Rauhocereus riosaniensis Backeberg & Cactaceae & $x$ & $x$ & $x$ & $x$ & $x$ & & $x$ & & \\
\hline 17 & Croton adipatus Kunth & Euphorbiaceae & & & & & & & & & $x$ \\
\hline 18 & Ditaxis dioica H. B. K. & Euphorbiaceae & & & $x$ & & $x$ & & & $x$ & \\
\hline 19 & Ditaxis katharinae Pax & Euphorbiaceae & $x$ & $x$ & & & & & $x$ & & \\
\hline 20 & Jatropha humboldtiana McVaugh & Euphorbiaceae & & & $x$ & $\mathrm{x}$ & & & & $x$ & \\
\hline 21 & Bauhinia suaveolens H. B. K. & Leguminosae & & & & & & & & & $\mathrm{x}$ \\
\hline 22 & Caesalpinia sp. nova* (JLMP-2040) & Leguminosae & & & & $\mathrm{x}$ & & & & $x$ & \\
\hline 23 & Calliandra mollissima (Humb. \& Bonpl. ex Willd.) Benth. & Leguminosae & $x$ & & & & & & $x$ & & \\
\hline 24 & Coursetia maraniona Lavin (JLMP-2004) & Leguminosae & & & & & & & & & $x$ \\
\hline 25 & Maraniona lavinii C.E. Hughes, G.P. Lewis, Daza \& Reynel & Leguminosae & & & & & & & & & $x$ \\
\hline 26 & Mimosa incarum Barneby & Leguminosae & & & & & & & & & $x$ \\
\hline 27 & Mimosa pectinatipinna Burkart & Leguminosae & & $x$ & & $x$ & & $\mathrm{x}$ & & $x$ & \\
\hline 28 & Mimosa weberbauerii Harms & Leguminosae & & & & $x$ & & & & & \\
\hline 29 & Tetrasida chachapoyensis (Baker f.) Fryxell \& Fuertes & Malvaceae & $\mathrm{x}$ & $x$ & $x$ & $x$ & $\mathrm{x}$ & & $x$ & $\mathrm{x}$ & \\
\hline 30 & Tetrasida serrulata Fryxell \& Fuertes & Malvaceae & & & & & & & & & $\mathrm{x}$ \\
\hline 31 & Ruprechtia aperta Pendry & Polygonaceae & $\mathrm{x}$ & $x$ & $x$ & $x$ & $\mathrm{x}$ & & $\mathrm{x}$ & $x$ & \\
\hline 32 & Esenbeckia cornuta Eng. & Rutaceae & & & & $x$ & & & & & \\
\hline
\end{tabular}

${ }^{\star}$ T. R. Pennington Y C. Hughes, Manifiestan Que Es Una Especie Nueva Para La Ciencia. 
de Tarapoto (Departamento de San Martín) y 55 especies (sin incluir lianas) para los BTES del departamento de Cajamarca. Cabe indicar que su estudio para el departamento de Cajamarca se restringió solo a colecciones generales que realizó durante sus recorridos a las parcelas de evaluación. Nuestro estudio incrementa notablemente los listados de Weberbaur, Ferreyra y Bridgewater et al. Así mismo, es más diverso en plantas leñosas que el Coto de Caza El Angolo.

Aguirre \& Kvist (2005) en sus estudios de los bosques secos del sur-occidente del Ecuador que incluyeron las provincias de Loja, El Oro, Guayas, Manabí, Esmeralda y Los Ríos, registraron 219 especies de plantas leñosas. Estos resultados son mayores que los nuestros, aunque nuestro estudio se limitó a un solo distrito (en Ecuador una provincia es equivalente a la Región).

Inventarios Botánicos Rápidos realizados en BTES de la costa del Pacífico de Honduras y la costa de Oaxaca, al sur de México; en áreas de tamaño variable $(40,3$ y $38,4 \mathrm{Ha})$ y con 4,5 horas de evaluación por muestra, Gordon et al. (2004) reportaron 194 especies de 22 áreas evaluadas para Honduras y 252 especies de 21 áreas evaluadas para Oaxaca-México. Estos resultados muestran una diversidad alta en contraste con lo observado en el presente estudio. Sin embargo, no se precisa cuantas especies fueron registradas en cada unidad muestral (de las 22 para Honduras y 21 para Oaxaca), sólo se presentan el total de las especies de toda la muestra. Así mismo, el área, el tiempo de evaluación y las brigadas de trabajo de campo fueron considerablemente mayores en Honduras y Oaxaca.

Linares-Palomino (2004), en su lista (http://rbg-web2.rbge. org.uk/dryforest/database.htm), incluye 181 especies para los BTES del Sistema del Marañón, de éstas, el 37\% (67 de las 181), también fueron registradas en nuestro estudio. Cabe destacar que esta investigación incrementa con 57 especies la lista de los BTES del sistema del Marañón. No obstante, este número se incrementará, cuando se complete la colección fértil y se concluya la determinación del material botánico. Debo indicar que para el presente análisis, no se consideró especies cultivadas o aquellas plantas leñosas que no superan los 2,0 m de altura.

Gentry (1995), menciona que Leguminosae y Bignoniaceae son las familias más dominantes y las que poseen mayor diversidad de especies de los BTES del neotrópico. En la zona de estudio Leguminosae también es la más diversa con 36 especies, le siguen Cactaceae y Euphorbiaceae con 16 especies. Marcelo-Peña y colaboradores (2007), en sus estudios de cuatro muestras de 0.1 ha en los BTES del distrito de Jaén, registraron a estas mismas familias como las más diversas. Sin embargo, las familias más dominantes fueron Boraginaceae, Leguminosae y Cactaceae. Bignoniaceae no es diversa ni dominante. Una especie de Tabebuia posee alta demanda como combustible (leña para cocina, panaderías y ladrilleras), y hace cuatro décadas fue talado para la construcción y la industria del parquet. Actualmente sus poblaciones son escasas.

Gentry (1995), indica que los géneros más comunes en los bosques secos del Neotrópico son Tabebuia, Casearia, Baubinia, Trichilia, Erythroxyllum, Randia, Hippocratea, Serjania, Croton y Zanthoxylum. Estos patrones de diversidad difieren con la zona de estudio, excepto por Croton y Serjania. No fueron registrados Casearia, Trichilia, Randia y Hippocratea. En este estudio los géneros más diversos y comunes son Croton, Acacia, Cordia, Capparis y Espostoa. Senna y Bauhinia son diversos. Sin embargo, no son comunes (poco frecuentes). Marcelo-Peńa y colaboradores (2007), registraron a Cordia, Tetrasida, Browningia, Croton, Praecereus y Ruprechtia, como los géneros más comunes $\mathrm{y}$ abundantes.

\section{De las especies endémicas}

León y colaboradores (2006) reportan un total de 948 especies endémicas de la región Cajamarca. Linares-Palomino (2006), reporta 96 especies endémicas para los BTES del Marañón. La zona representa el 3,3 \% de taxones endémicos de la región Cajamarca y el 33,3\% de las especies endémicas de los BTES del Marañón. Los niveles altos de endemismo de los BTES de Jaén se pueden atribuir al carácter fragmentado de este hábitat y a su aislamiento geográfico que lo inducen a la especiación (Hensold, 1999).

Un hallazgo importante es el registro de $M$. lavinii, único género endémico de los BTES de Jaén, recientemente descubierto por C. C Hughes et al. (2004), en la zona de Balsas (límite entre Cajamarca y Amazonas), en donde es frecuente. Sin embargo, en la zona de estudio solo se registró un individuo en los alrededores de Shanango y tres individuos en los bosques del Pongo al noreste de la ciudad de Jaén (zona recientemente evaluada por T. Sarkiten). Hoy el distrito de Jaén es el límite de su distribución natural al noroeste de la región Cajamarca.

O. cabrerae, C. tenuiserpens, P. horrida ssp. rauhii, C. adipatus, $B$. suaveolens, C. maraniona, $M$. lavinii, $M$. incarum y T. serrulata, no fueron registradas durante los IBR, pero se los encontró en los recorridos a las zonas de evaluación. Por ejemplo, $O$. cabrerae es rara en San Isidro1, $C$. tenuiserpens, es rara en San Isidro1 y San Isidro2, P. horrida spp. raubii, se encuentra dispersa en el sector de Mochenta. C. adipatus se encuentra esparcida en Mochenta, San Isidro1 y el Almendral.

$B$. suaveolens, es rara en el Almendral, $M$. incarum es rara en El Huito y $T$. serrulata es rara en San Isidro2 y Mochenta

En las suculentas Hylocereus microcladus es endémica restringida sólo a los BTES del distrito de Jaén, esta especie fue citada en 1958 por Rauh y no ha sido discutida en otros estudios taxonómicos de la Familia Cactaceae por carecer de material botánico. C. tenuiserpens y E. blossfeldiorum, son endémicas del Norte del Perú. Todas las demás son endémicas restringidas a los BTES del Marañón. Taylor, citado por Bridgewater et al. (2003), indica que la región del Valle del Marañón es rica en cactáceas endémicas, la cual pueda reflejar que es cercana al área ancestral de las cactáceas.

De las leñosas, son especies endémicas restringidas solo a los BTES del distrito de Jaén $D$. katharinae y E. cornuta. Son especies endémicas del Norte del Perú $C$. adipatus y $B$. suaveolens. Onoseris cabrerae posee poblaciones en el norte y el sur del Perú (Huancavelica, Apurímac y Cuzco), 16 especies son endémicas restringidas a los BTES del Marañón.

Como registro interesante de los sectores estudiados destacó el hallazgo de E. cornuta, que no ha sido reportada en 156 años, desde la colección tipo realizada por Warscewicz en 1851 . Existen algunas diferencias morfológicas entre el material botánico que actualmente preservo y la descripción de E. cornuta proporcionada en Flora del Perú (Macbride, 1949). Las diferencias se observan en la longitud del pecíolo y del fruto. La clave indica que los pecíolos son 2 a 2,5 cm de largo, sin embargo, los pecíolos observando el isotipo en línea (http://sweetgum. 
nybg.org/common/imagedisplay.php?irn=42650), miden entre 1-2 cm. Las muestras colectadas en Shanango poseen frutos más largos con respecto a la descripción original, debido a que éstos fueron colectados cuando estaban en desarrollo y las medidas corresponden a frutos juveniles. Es necesario elaborar una descripción más precisa y detallada de la especie ahora que se posee material botánico completo.

León et al. (2006), no le otorgó a E. cornuata una categoría de amenaza por presentar datos insuficientes. Sin embargo, puedo considerarla como una especie en peligro crítico de extinción, por su rango geográfico restringido solo al sector de Shanango (con un área de ocupación menor a $10 \mathrm{~km}^{2}$ ), por la severa fragmentación de su hábitat y la eminente desaparición de sus poblaciones por la actividad agrícola y ganadera. Como ejemplo de esa amenaza podemos mencionar que durante el periodo de estudio a aproximadamente unos $400 \mathrm{~m}$ de la población evaluada, se destruyó el bosque para usar el terreno para el cultivo de maíz.

León et al. (2006) en El Libro Rojo de las Plantas endémicas del Perú, no consideró a Monactis rhombifolia, Ditaxis katharinae, Bauhinia suaveolens y Jatropha humboldtiana, que según Brako \& Zarucchi (1993), fueron incluidas como endémicas para nuestro país.

Aguirre \& Kvist (2005), en los bosques secos del Sur Occidente del Ecuador que incluye las provincias de Loja, El Oro, Guayas, Manabí, Esmeralda y Los Ríos, registraron 15 especies endémicas. Gordon et al. (2004) reportaron 11 especies endémicas en la costa del Pacífico de Honduras y la costa de Oaxaca al Sur de México. En contraste, los BTES del distrito de Jaén presentan valores sorprendentemente altos en endemismos, es 17 especies mayor en comparación con los bosques del Sur Occidente de Ecuador y 21 especies mayor comparado con los bosques de Honduras y Oaxaca.

Todos estos registros han empezado a revelar las características únicas de los BTES del distrito de Jaén, que desafortunadamente están altamente amenazados. Por lo tanto, es de suma importancia iniciar Programas de Manejo y Conservación de la flora de la provincia de Jaén.

\section{De la influencia antrópica}

En la provincia de Jaén las especies comerciales de los BTES fueron extraídas hace aproximadamente cuatro décadas, Tabebuia chrysantha "Guayacán" y Aspidosperma polyneuron "acerillo" se talaron para la construcción de viviendas (utilizados para la confección de columnas, vigas, tijerales, dinteles) y en las últimas décadas para la industria del parquet. Actualmente ya no quedan individuos maduros de buen porte y diámetro comercial. Sus poblaciones se han reducido a individuos juveniles.

Las áreas de BTES que hoy presentan suministro de riego han sido talados y en ellas se han instalado cultivos de "arroz" (Montegrande, Fila Alta, Linderos, Yanayacu, Shumba, Santa Cruz y Bellavista) o se han instalado plantaciones agroforestales de Theobroma cacao "Cacao", Musa sp., "plátano", Cocus nucifera L. "coco" e Inga spp. "guaba"; frutales como Mangifera indica L. "mango", Spondias purpurea L. "ciruela”, Citrus spp. "naranjas”, "limones", Carica papaya L. "papaya”, o cultivos semestrales de M. sculenta "yuca", Zea maiz "maíz", Phaseolus vulgaris L. "frejol" o Glycine max (L.) Merr. "soya”.
Las colinas del Suroeste y Oeste de la ciudad de Jaén están casi completamente desprovistas de vegetación, la parcelación a traído como consecuencia su tala progresiva y a facilitado la instalación de cultivos semestrales de $Z$. maiz "maíz", M. sculenta "yuca" y Phaseolus spp. "frejol", para el auto consumo o para el mercado local. Sin embargo, estos cultivos posteriormente, dan paso a los sembríos de pastos para mantener ganadería extensiva y durante la época seca cuando los pastos pierden vigor, para "recuperarlos" los propietarios de los pastizales inician la quema, que ocasiona la pérdida de la regeneración natural y de otros elementos de la flora y fauna del suelo. Estas actividades incrementan el deterioro del suelo, dando paso a una formación tipo sabana con especies arbóreas de Cybistax antisyphilitica (C. Martius) C. Martius ex A. DC., Luehea paniculada Mart \& Zucc., Aspidosperma polyneuron "acerillo", Acacia macracantha "faique o huarango", Cordia iguaguana "iguaguana", Hura crepitans "catagua", Sapindus saponaria L. "choloque", Capparis scabrida Kunth "zapote o zapotillo", Pseudobombax sp. entre las más conspicuas y especies arbustivas de Boungainvillea peruviana, Malpighia glabra L., Cyathostegia matthewsii, Tournefortia hirsutissima L.

Actualmente quedan pequeños relictos de vegetación estacionalmente seca, al oeste y Noroeste, el más representativo, es el que se encuentra en el sector El Huito, con un área aproximada de 15 ha, seriamente amenazado por la demanda de especies leñosas combustibles y la ganadería extensiva.

Al Este, Sureste y Noreste de la ciudad de Jaén, el BTES es más amplio. Sin embargo, las trochas, las carreteras, las invasiones, la creciente venta de solares para la construcción de viviendas y la demanda de especies de uso combustible, están fragmentando completamente el paisaje y se han convertido en una amenaza latente para la flora del BTES de Jaén.

Si seguimos el enfoque global para determinar la importancia de la diversidad con prioridad de conservación, el distrito de Jaén cuenta con 32 especies endémicas con rangos de distribución restringidos y con alto grado de extinción. Tomando como referencia este criterio, Mochenta, Shanango, San Isidro1 y San Isidro2, son los sectores identificados con prioridad de conservación, por presentar mayores niveles de endemismos y considerarlas como muestras representativas de la vegetación leñosas de los BTES de Jaén.

\section{Conclusiones}

Los BTES del distrito de Jaén muestran menor diversidad que los bosques tropicales húmedos. Sin embargo, presentan mayor diversidad que los BTES de regiones vecinas. Las familias más comunes y diversas son Leguminosae, Cactaceae y Euphorbiaceae. Los géneros más diversos y comunes son Croton, Acacia, Cordia, Capparis y Espostoa.

Los BTES del distrito de Jaén destacan por sus altos niveles de endemismos. La zona presenta valores sorprendentemente altos en contraste con los BTES interandinos y orientales de nuestro país y los BTES del Sur Occidente del Ecuador.

Estos registros nos revelan las características únicas de los BTES del distrito de Jaén, que desafortunadamente están seriamente amenazados, por las poblaciones que demandan de especies para material combustible, áreas para cultivos agrícolas, ganadería extensiva y vivienda. 
Propongo los sectores de Mochenta, Shanango, San Isidro 1 y San Isidro 2 con prioridad de conservación. Si estas áreas son de propiedad privada o son de libre disponibilidad, debemos concentrar nuestro esfuerzo por crear áreas de conservación privada o áreas de conservación municipal, que deberán ser conducidos con Programas de manejo y conservación de la flora de la provincia de Jaén.

\section{Agradecimientos}

El autor agradece a T. Pennington y Reynaldo Linares por sus comentarios al manuscrito. A C. Ostolaza por la determinación de las cactáceas. J. Albán por las facilidades brindadas en el Herbario USM. A mis colegas J. Millán, J. Gonzales y D. Elera por su apoyo en el trabajo de campo y a F. Arroyo, A. Pérez de la Universidad Nacional Agraria La Molina por su colaboración en el digitado de las etiquetas y montaje de las muestras botánicas.

\section{Literatura citada}

Aguirre, M. A. \& L. P. Kvist. 2005. Composición florística y estado de conservación de los bosques secos del Sur-Occidente del Ecuador. Lyonia 8 (2): 41-67

INRENA, 1995. Mapa Forestal del Perú escala 1:1 000000 con guía explicativa. Lima, Instituto Nacional de Recursos Naturales. Perú.

Ediciones Peisa, 2004. Atlas regional del Perú. Tomo 13: Cajamarca. Imagen geográfica, estadística, histórica y cultural. Ediciones Peisa. Perú. Pp. 103.

Barneby, R. C. \& J. W. Grimes. 1997. Silk Tree, Guanacaste, Monkey's Earring. A generic system fro the synandrous Mimosaceae of the Americas. Part II: Pithecellobium, Cojoba \& Zygia. Mem. New York Bot. Gard. 74 (2): 1-149

Berg, C. C. \& S. V. Dahlberg (2001). A revision of Celtis subg. Mertensia (Ulmaceae). Brittonia 53: 66-81

Brako, L. \& J. Zarucchi. 1993. Catálogo de las Angiospermas y Gimnospermas del Perú. Monogr. Syst. Bot. Missouri Bot. Garden. Vol $45: 1-1286$

Bridgewater, S.; R. T. Pennington; C. Reynel; A. Daza \& T. D. Pennington. 2003. A preliminary floristic and phytogeographic analysis of the woody flora of seasonally dry forest in northern Peru. Candollea 58: 129-148

Ferreyra, R. 1996. Comunidades vegetales de la cuenca superior de los ríos: Marañón, Huallaga y Ucayali. Documento Técnico $\mathrm{N}^{\circ}$ 27. IIAP. Iquitos, Perú.

Gentry, A. H. 1995. Diversity and floristic composition of Neotropical dry forests. In: S. H. Bullock; H. A. Money \& E. Medina (Eds.) Seasonally Dry Tropical Forests. Cambridge, Cambridge University Press. pp. 146-194.

Gordon J. E.; W. D. Hawthorne; A. Reyes-García; G. Sandoval \& A. J. Barrance. 2004. Assesing landscapes: a case study of tree and shrub diversity in the seasonally dry tropical fortest of Oaxaca, Mexico and southern Honduras. Biological conservation 117: 429-442.

Hawthorne, W. D. \& M. Abu-Juam. 1995. Forest Protection in ghana. With particular reference vegetation and plant species. Forest inventory and Management Project Planning Brach, Forestry Deparment Kumasi, Ghana. IUCN/ODA/Forest Deparment Republic of Ghana.
Hensold, N. 1999. Las Angiospermas endémicas del Departamento de Cajamarca, Perú. Arnaldoa 6(2): 141-184.

León, B., J. Roque, C. Ulloa, N. Pitman, P.M. Jorgensen \& A. Cano (eds.) 2006. El libro Rojo de las Plantas Endémicas del Perú. Revista Peruana de Biología. Número especial 13(2). 1-967

Linares-Palomino, R. 2004. Los Bosques Tropicales Estacionalmente Secos: II. Fitogeografía y Composición Florística. Arnaldoa 11(1):103-138

Linares-Palomino R. 2006. Phytogeography and Floristics of Seasonally Dry Forests in Peru. En Pennington, R. T., Lewis, G. P. \& Ratter, J. A. (eds.) Neotropical savannas and Seasonally Dry Forests: Plant Diversity, Biogeography and conservation. CRC, Boca Raton, FL.pp. 257-279.

Macbridei, J. F. 1949. Flora of Peru. Botanical Series. Field Museum of Natural History. Volume XIII, Part III. Number 2. Publication 622. pp 511-777

Marcelo-Peña, J. L.; C. Reynel; P. Zevalllos; F. Bulnes \& A. Ojeda. 2007. Diversidad, composición florística y endemismos de la vegetación leñosa en los bosques estacionalmente secos alterados del distrito de Jaén, Perú. Rev. Ecol. Aplic. $6(1,2)$

Murphy, P. \& A. E. Lugo. 1995. Dry forest of Central America and tha Caribbean. In: Bullock, S.H.; H.A.Mooney / E. Medina (eds.), Seasonally dry tropical forests: 9-34. Cambridge University Press.

Pendry, C. A. 2003. Nine new species of Ruprechtia (Polygonaceae) from Central y South America. Edinburgh Journal of Botany 60(1): 19-42

Pennington, R. T.; D. E. Prado; C. A. Pendry. 2000 Neotropical Seasonally dry forests and quaternary vegetation changes. J. Biogeog. 27: 261-273.

Ratter, J.A.; G.P. Askew; R. Montgomery \& D.R. Gifford. 1978. Observations on forests of some meso-trophic soils in central Brazil. Revista Brasil . Bot. I: 47-58.

Ríos T., J. 1989. Análisis del hábitat del Coto de Caza El Angolo - Piura. Escuela de Post-Grado. Tesis (Mg Sc). Universidad Nacional Agraria La Molina, Lima, Perú. 268 p.

Rodríguez, R. E. \& Rojas, G. R. 2002. El Herbario. Administración y manejo de colecciones botánicas. Editado por R. Vásquez M. Jardín Botánico de Missouri - PERÚ.

Sagástegui A., M. O. Dillon, I. Sánchez, S. Leiva \& P. Lezama. 1999. Diversidad florística del Norte del Perú. Tomo I. WWF \& Fondo Editorial Universidad Privada Antenor Orrego. Trujillo, Perú.

Valencia, R., N. Pitman, S. León-Yanez \& P. M. Jorgensen (eds.) 2000. Libro de las plantas endémicas del Ecuador 2000. Herbario QCA, Pontificia Universidad Católica del Ecuador, Quito. Pp 489.

Weberbauer, A. 1945. El mundo vegetal de los Andes Peruanos. Estac. Exper. Agric. La Molina. Edit. Lume. Lima. Pp: 776

Whitmore, T. C. 1998. An introduction to Tropical Rain Forests. Oxford University Press. Pp: 296 
Apéndice 1. Plantas leñosas registradas en los bosques tropicales estacionalmente secos (BTES) del distrito de Jaén, Cajamarca, Perú. A. Sector El Huito; B. Sector San Isidro1; C. Sector San Isidro2; D. Sector Shanango; E. Sector Yanuyacu; F. Sector El Almendral; G. Sector Linderos Alto; H. Sector Mochenta; Al. Alrededores de las áreas evaluadas.

\begin{tabular}{|c|c|c|c|c|c|c|c|c|c|c|c|}
\hline $\mathbf{N}^{\circ}$ & Género y especie & Familia & A & B & $\mathrm{C}$ & D & E & $\mathbf{F}$ & G & $\mathbf{H}$ & Al. \\
\hline 1 & Aphelandra glabra Willd. ex Nees & Acanthaceae & $\mathrm{x}$ & & & & & & & & \\
\hline 2 & Achatocarpus pubescens C. H. Wringht & Achatocarpaceae & & & & & & & & & $\mathrm{x}$ \\
\hline 3 & Annona sp. (JLMP-1987) & Anonnaceae & $\mathrm{x}$ & & & & & & & & \\
\hline 4 & Annona cherimolia Mill. & Anonnaceae & & & & & & & & & $x$ \\
\hline 5 & Annona muricata L. & Anonnaceae & & & & & & & & & $\mathrm{x}$ \\
\hline 6 & Aspidosperma polyneuron Müll. Arg. & Apocynaceae & $\mathrm{x}$ & $\mathrm{x}$ & $\mathrm{x}$ & & $\mathrm{x}$ & & $\mathrm{x}$ & & \\
\hline 7 & Vallesia glabra (Cav.) Link & Apocynaceae & & & & & & $\mathrm{x}$ & & $\mathrm{x}$ & \\
\hline 8 & Monactis rhombifolia Sagast. \& Dillon & Asteraceae & $\mathrm{x}$ & $\mathrm{x}$ & $x$ & $x$ & & & $\mathrm{x}$ & & \\
\hline 9 & Onoseris cabrerae Ferreyra & Asteraceae & & & & & & & & & $\mathrm{x}$ \\
\hline 10 & Tessaria integrifoliaRuiz \& Pav. & Asteraceae & & & & & & & & & $x$ \\
\hline 11 & Vernonanthura patens (Kunth) H. Rob. & Asteraceae & & & & & & & & & $x$ \\
\hline 12 & Baccharis sp. & Asteraceae & & $\mathrm{x}$ & & $\mathrm{x}$ & & & & & $x$ \\
\hline 13 & Crescentia cujete L. & Bignoniaceae & & & & & & & & & $x$ \\
\hline 14 & Cybistax antisyphilitica (C. Martius) C. Martius ex A. DC. & Bignoniaceae & & & & & & & & & $x$ \\
\hline 15 & Tabebuia chrysantha subsp. meridionalis A. H. Gentry & Bignoniaceae & $\mathrm{x}$ & $\mathrm{x}$ & $\mathrm{x}$ & $\mathrm{x}$ & $\mathrm{x}$ & & $\mathrm{x}$ & $\mathrm{x}$ & \\
\hline 16 & Tecoma rosaefolia Kunth & Bignoniaceae & & & & & & & & $\mathrm{x}$ & \\
\hline 17 & Tecoma stans (L.) Juss. ex Kunth & Bignoniaceae & & & & & & & & & $x$ \\
\hline 18 & Ceiba insignis (Kunth) P. E. Gibbs \& Semir & Bombacaceae & $\mathrm{x}$ & $\mathrm{x}$ & $\mathrm{x}$ & $\mathrm{x}$ & $\mathrm{x}$ & & $\mathrm{x}$ & $\mathrm{x}$ & \\
\hline 19 & Eriotheca discolor (Kunth) A. Robyns & Bombacaceae & & $\mathrm{x}$ & $\mathrm{x}$ & $\mathrm{x}$ & & $\mathrm{x}$ & $x$ & $\mathrm{x}$ & \\
\hline 20 & Ochroma pyramidale (Cav. ex Lam.) Urb. & Bombacaceae & & & & & & & & & $\mathrm{x}$ \\
\hline 21 & Pseudobombax sp. (JLMP-1988) & Bombacaceae & $\mathrm{x}$ & & & & & & & & \\
\hline 22 & Cordia iguaguana Melch. ex I.M. Johnst. & Boraginaceae & $x$ & $\mathrm{x}$ & $x$ & $\mathrm{x}$ & $\mathrm{x}$ & & $\mathrm{x}$ & & \\
\hline 23 & Cordia lutea Lam. & Boraginaceae & & & & & & & & $\mathrm{x}$ & \\
\hline 24 & Cordia saccellia Gottschling \& J.S. Mill. & Boraginaceae & $\mathrm{x}$ & $\mathrm{x}$ & $\mathrm{x}$ & $\mathrm{x}$ & $\mathrm{x}$ & $\mathrm{x}$ & $\mathrm{x}$ & $\mathrm{x}$ & \\
\hline 25 & Cordia varronifolia J. M. Johnson & Boraginaceae & & $\mathrm{x}$ & & & & & & $x$ & \\
\hline 26 & Cordia macrocephala (Desv.) Kunth & Boraginaceae & & & & & & & & & $\mathrm{x}$ \\
\hline 27 & Heliotropium procumbens Mill. (JLMP-2020) & Boraginaceae & & & $\mathrm{x}$ & & & & & & \\
\hline 28 & Tournefortia hirsutissima $\mathrm{L}$. & Boraginaceae & & & & & $x$ & & & & \\
\hline 29 & Tournefortia sp. & Boraginaceae & & & $x$ & & & & & & \\
\hline 30 & Armatocereus rahuii Backeb. & Cactaceae & & & & & $\mathrm{x}$ & $\mathrm{x}$ & & $\mathrm{x}$ & \\
\hline 31 & Browningia altissima (F. Ritter) Buxb. & Cactaceae & $x$ & $x$ & $\mathrm{x}$ & $\mathrm{x}$ & $x$ & $x$ & $x$ & $x$ & \\
\hline 32 & Cleistocactus tenuiserpens Rauh \& Backeb. & Cactaceae & & & & & & & & & $\mathrm{x}$ \\
\hline 33 & Ephiphyllum phyllanthus (L.) Haw. & Cactaceae & & & & & & & & & $x$ \\
\hline 34 & Espostoa blossfeldiorum (Werdermann) Buxbaum & Cactaceae & & & & $\mathrm{x}$ & $\mathrm{x}$ & $\mathrm{x}$ & & & \\
\hline 35 & Espostoa lanata (Kunth) Britton \& Rose & Cactaceae & & $\mathrm{x}$ & $x$ & $x$ & & $x$ & $\mathrm{x}$ & $x$ & \\
\hline 36 & Espostoa mirabilis F. Ritte & Cactaceae & & & & & & & & $\mathrm{x}$ & \\
\hline 37 & Espostoa superba F. Ritter & Cactaceae & & & $\mathrm{x}$ & & & & & & \\
\hline 38 & Hylocereus microcladus Backeberg & Cactaceae & $\mathrm{x}$ & & & & & & & & \\
\hline 39 & Melocactus bellavistensis Rauh \& Backeberg & Cactaceae & & $x$ & & & & $\mathrm{x}$ & $\mathrm{x}$ & $\mathrm{x}$ & \\
\hline 40 & Praecereus euchlorus subsp. jaenensis (Rauh \& Backeb.) Ostolaza & Cactaceae & $\mathrm{x}$ & $\mathrm{x}$ & $\mathrm{x}$ & $\mathrm{x}$ & $\mathrm{x}$ & $\mathrm{x}$ & $\mathrm{x}$ & $\mathrm{x}$ & \\
\hline 41 & Opuntia macbridei Britton \& Rose & Cactaceae & & $\mathrm{x}$ & $\mathrm{x}$ & & $\mathrm{x}$ & $\mathrm{x}$ & & $\mathrm{x}$ & \\
\hline 42 & Pereskia horrida (Kunth) DC. & Cactaceae & & & $\mathrm{x}$ & & $\mathrm{x}$ & $\mathrm{x}$ & $\mathrm{x}$ & $\mathrm{x}$ & \\
\hline 43 & Pereskia horrida ssp. Rauhi (Backeberg) Ostolaza & Cactaceae & & & & & & & & & $\mathrm{x}$ \\
\hline 44 & Rauhocereus riosaniensis Backeberg & Cactaceae & $\mathrm{x}$ & $\mathrm{x}$ & $x$ & $x$ & & $\mathrm{x}$ & $\mathrm{x}$ & & \\
\hline 45 & Capparis crotonoide Kunth & Capparidaceae & $\mathrm{x}$ & & & & & $x$ & & $\mathrm{x}$ & \\
\hline 46 & Capparis flexuosa (L.) L. & Capparidaceae & & $x$ & $\mathrm{x}$ & $\mathrm{x}$ & $\mathrm{x}$ & $\mathrm{x}$ & $\mathrm{x}$ & $\mathrm{x}$ & \\
\hline 47 & Capparis prisca J.F. Macbr. & Capparidaceae & $\mathrm{x}$ & & & & & & & & \\
\hline 48 & Capparis scabrida Kunth & Capparidaceae & $\mathrm{x}$ & $\mathrm{x}$ & & & $\mathrm{x}$ & $\mathrm{x}$ & $\mathrm{x}$ & $\mathrm{x}$ & \\
\hline 49 & Maytenus octogona (L' Her.) DC. & Celastraceae & & $x$ & $\mathrm{x}$ & & $x$ & & & $\mathrm{x}$ & \\
\hline 50 & Clusia sp. (JLMP-1776) & Clusiaceae & $x$ & & $x$ & & $x$ & & $\mathrm{x}$ & & \\
\hline 51 & Ipomoea carnea Jacq. & Convolvulaceae & & & & & $\mathrm{x}$ & $\mathrm{x}$ & & & \\
\hline 52 & Jacquemontia floribunda (Kunth) Hallier f. & Convolvulaceae & & & & & & & & $x$ & \\
\hline 53 & Erythroxylum impresum O. E. Schulz aff. & Erythroxylaceae & $x$ & $\mathrm{x}$ & $x$ & & & $x$ & & & \\
\hline 54 & Acalypha sp. (JLMP-2251) & Euphorbiaceae & & & $\mathrm{x}$ & & & & & & \\
\hline 55 & Acalypha sp. (JLMP-2255) & Euphorbiaceae & & & & & & & & & $\mathrm{x}$ \\
\hline 56 & Cnidoscolus jaenensis (Pax \& K. Hoffm.) J.F. Macbr. & Euphorbiaceae & & $\mathrm{x}$ & & & $\mathrm{x}$ & & $x$ & & \\
\hline 57 & Croton adipatus Kunth & Euphorbiaceae & & & & & & & & & $\mathrm{x}$ \\
\hline
\end{tabular}


Apéndice 1. (continuación)

\begin{tabular}{|c|c|c|c|c|c|c|c|c|c|c|c|}
\hline $\mathbf{N}^{\circ}$ & Género y especie & Familia & $\mathbf{A}$ & B & $\mathrm{C}$ & $\mathbf{D}$ & E & $\mathbf{F}$ & G & $\mathbf{H}$ & Al. \\
\hline 58 & Croton pedicellatus Kunth & Euphorbiaceae & & & & & & & & & $x$ \\
\hline 59 & Croton pycnanthus Benth. & Euphorbiaceae & & & & & & & & & $x$ \\
\hline 60 & Croton thurifer Kunth & Euphorbiaceae & & & $x$ & $x$ & $x$ & $x$ & $x$ & $x$ & \\
\hline 61 & Ditaxis dioica H. B. K. & Euphorbiaceae & & & $x$ & & $x$ & & & $x$ & \\
\hline 62 & Ditaxis katharinae Pax & Euphorbiaceae & $x$ & $x$ & $x$ & & & & $x$ & & \\
\hline 63 & Hura crepitans $\mathrm{L}$. & Euphorbiaceae & $x$ & & $x$ & & $x$ & & & & \\
\hline 64 & Jatropha humboldtiana McVaugh & Euphorbiaceae & & & $x$ & $x$ & & & & $x$ & \\
\hline 65 & Jatropha clavulifera Müll. Arg. & Euphorbiaceae & & & & & & & & & $x$ \\
\hline 66 & Phenax laevigatus Weddell & Euphorbiaceae & & & & & & & & & $x$ \\
\hline 67 & Ricinus communis $\mathrm{L}$. & Euphorbiaceae & & & & & & & & & $x$ \\
\hline 68 & Sebastiana sp. (JLMP-2246) & Euphorbiaceae & & & & & & $\mathrm{x}$ & & & \\
\hline 69 & Krameria lappacea (Dombey) Burdet \& B. Simpson & Krameriaceae & & & & & & $x$ & & & \\
\hline 70 & Acacia aroma Gillies ex Hook. \& Arn. & Leguminosae & & & & & & & $x$ & $x$ & \\
\hline 71 & Acacia macracantha Humb. \& Bonpl. ex Willd. & Leguminosae & $\mathrm{x}$ & $\mathrm{x}$ & $x$ & $x$ & $x$ & $\mathrm{x}$ & $x$ & $x$ & \\
\hline 72 & Acacia polyphylla DC. & Leguminosae & & & & & & & & & \\
\hline 73 & Acacia riparia H. B. К. & Leguminosae & $x$ & $x$ & $x$ & & $x$ & & $x$ & $x$ & \\
\hline 74 & Acacia weberbaueri Harms & Leguminosae & & $x$ & $x$ & $x$ & $\mathrm{x}$ & & $x$ & $x$ & \\
\hline 75 & Albizia multiflora (Kunth) Barneby \& J.W. Grimes var. multiflora & Leguminosae & $x$ & & & & $x$ & & $x$ & $x$ & \\
\hline 76 & Albizia niopoides (Spruce ex Benth.) Burkart & Leguminosae & & & & & & & & & \\
\hline 77 & Anadenanthera colubrina (Vell.) Brenan & Leguminosae & $x$ & & & & & & & $x$ & \\
\hline 78 & Bauhinia augusti Harms & Leguminosae & & & & & & & & $x$ & \\
\hline 79 & Bauhinia suaveolens H. B. K. & Leguminosae & & & & & & & & & $x$ \\
\hline 80 & Bauhinia glabra Jacq. & Leguminosae & $x$ & & & & & & & & \\
\hline 81 & Bauhinia sp. & Leguminosae & & & & & & & & & $x$ \\
\hline 82 & Caesalpinia cassioides Willd. & Leguminosae & & $\mathrm{x}$ & $x$ & & & & $x$ & $x$ & \\
\hline 83 & Caesalpinia sp. nova (JLMP-2040) & Leguminosae & & & & $x$ & & & & $x$ & \\
\hline 84 & Calliandra mollissima (Humb. \& Bonpl. ex Willd.) Benth. & Leguminosae & $\mathrm{x}$ & & & & & & $\mathrm{x}$ & & \\
\hline 85 & Coursetia maraniona Lavin & Leguminosae & & & & & & & & & $x$ \\
\hline 86 & Cyathostegia matthewsii (Benth.) Schery & Leguminosae & $x$ & $x$ & $x$ & $x$ & $\mathrm{x}$ & & $x$ & $x$ & \\
\hline 87 & Dalea carthagenensis (Jacq.) Macbride var. brevis (Macbride) Barneby & Leguminosae & & & & $x$ & & & & & $x$ \\
\hline 88 & Erythrina amazónica Krukoff & Leguminosae & & & & & & & & & $x$ \\
\hline 89 & Geoffroea spinosa Jacq. (JLMP-2249) & Leguminosae & & & & & & & & & $x$ \\
\hline 90 & Indigofera suffruticosa Mill. & Leguminosae & & & & $x$ & & & & & $x$ \\
\hline 91 & Leucaena trichodes (Jacq.) Benth. & Leguminosae & $x$ & $x$ & $x$ & $x$ & $x$ & $x$ & $x$ & $x$ & \\
\hline 92 & Maranonia lavinii C.E. Hughes, G.P. Lewis, Daza \& Reynel & Leguminosae & & & & & & & & & $x$ \\
\hline 93 & Mimosa incarum Barneby & Leguminosae & & & & & & & & & $x$ \\
\hline 94 & Mimosa pectinatipinna Burkart & Leguminosae & & $x$ & & $x$ & & $x$ & & $x$ & \\
\hline 95 & Mimosa weberbaueri Harms & Leguminosae & & & & $x$ & & & & & $x$ \\
\hline 96 & Parkinsonia praecox (R. \& P.) Harms & Leguminosae & & & & & $x$ & $x$ & & $\mathrm{x}$ & \\
\hline 97 & Phitecellobium excelsum (Kunth) Benth. & Leguminosae & $x$ & $x$ & $x$ & $x$ & $x$ & $x$ & $x$ & $x$ & \\
\hline 98 & Platymiscium pinnatum (Jacq.) Dugand & Leguminosae & $x$ & & & & & & $x$ & & \\
\hline 99 & Prosopis juliflora (Sw.) DC. & Leguminosae & & & & & $x$ & $\mathrm{x}$ & & $x$ & \\
\hline 100 & Senna bicapsularis (L.) Roxb. var. bicapsularis (Harms) I. \& B. & Leguminosae & & & & & & & & & $x$ \\
\hline 101 & Senna galegifolia (L.) Barneby \& Lourteiz & Leguminosae & & $x$ & $x$ & & & & & & \\
\hline 102 & Senna hirsuta (L.) H. S. Irwin \& Barneby & Leguminosae & & & & & & & & & $x$ \\
\hline 103 & Senna macranthera (DC. ex Collad.) H. S. Irwin \& Barneby & Leguminosae & & & & & & & & & $x$ \\
\hline 104 & Senna mollisima (Willd.) Irwin \& Barneby var. mollissima & Leguminosae & & $x$ & & $x$ & $x$ & & & & \\
\hline 105 & Senna obtusifolia (L.) Irwin \& Barneby & Leguminosae & & & & & & & & & $x$ \\
\hline 106 & Adenaria floribunda Kunth & Lytraceae & & & & & & & & & $x$ \\
\hline 107 & Malpighia glabra L. & Malpighiaceae & $x$ & $x$ & $x$ & $x$ & & & & $x$ & \\
\hline 108 & Heteropteris sp. & Malpighiaceae & & & & & & & & & $x$ \\
\hline 109 & Tetrapteris sp. & Malpighiaceae & & & & & & & & $x$ & \\
\hline 110 & Tetrasida chachapoyensis (Baker f.) Fryxell \& Fuertes & Malvaceae & $x$ & $x$ & $x$ & $x$ & $x$ & & $x$ & $x$ & \\
\hline 111 & Tetrasida weberbaueri (Ulbr.) Fryxell \& Fuertes & Malvaceae & & & & & & & & & $x$ \\
\hline 112 & Abutilon sp. & Malvaceae & & & & & & & & & $x$ \\
\hline 113 & Abutilon pedunculare H. B. K. & Malvaceae & & & & & & & & & $x$ \\
\hline 114 & Ficus insipida Willd. & Moraceae & & & & & & & & & $x$ \\
\hline 115 & Maclura tinctorea (L.) Steud. & Moraceae & $x$ & $x$ & & & & & & & \\
\hline \multirow[t]{2}{*}{116} & Psidium guajavaL. & Myrtaceae & & & & & & & & & $x$ \\
\hline & & & & & & & & & cont & & \\
\hline
\end{tabular}


Apéndice 1. (continuación)

\begin{tabular}{|c|c|c|c|c|c|c|c|c|c|c|c|}
\hline $\mathbf{N}^{\circ}$ & Género y especie & Familia & A & B & $\mathrm{C}$ & D & $\mathbf{E}$ & $\mathbf{F}$ & G & $\mathbf{H}$ & Al. \\
\hline 117 & Bougainvillea peruvianaHumb. \& Bonpl. & Nyctaginaceae & & & $\mathrm{x}$ & $x$ & $\mathrm{x}$ & $x$ & & $\mathrm{x}$ & \\
\hline 118 & Bougainvillea pachiphylla Heimerl ex Standley & Nyctaginaceae & & & & & & & & & \\
\hline 119 & Chionanthus sp. & Oleaceae & $\mathrm{x}$ & $\mathrm{x}$ & $x$ & $x$ & $x$ & & $x$ & & \\
\hline 120 & Agonandra excelsa Griseb. & Opilaceae & & $\mathrm{x}$ & & & & & $\mathrm{x}$ & $\mathrm{x}$ & \\
\hline 121 & Oxalis sp. (JLMP-2268) & Oxalidaceae & & & & & & & & & $x$ \\
\hline 122 & Saguieria aculeata Jacq. & Phytolaccaceae & $\mathrm{x}$ & & $x$ & & & & & & \\
\hline 123 & Piper angustifolium Lam. & Piperaceae & & & & & & & & & $\mathrm{x}$ \\
\hline 124 & Piper sp. (JLMP-2604) & Piperaceae & & & $\mathrm{x}$ & & & & & & \\
\hline 125 & Ruprechtia aperta Pendry & Polygonaceae & $\mathrm{x}$ & $\mathrm{x}$ & $x$ & $x$ & $x$ & & $\mathrm{x}$ & $\mathrm{x}$ & \\
\hline 126 & Triplaris cumingiana Fisch. \& C.A. Mey. ex C.A. Mey. & Polygonaceae & $x$ & & & & & & & & \\
\hline 127 & Randia armata (Sw.) DC. & Rubiaceae & $\mathrm{x}$ & & & & & & & & \\
\hline 128 & Esenbeckia cornuta Eng. & Rutaceae & & & & $x$ & & & & & \\
\hline 129 & Zanthoxylum fagara var. culantrillo (Kunth) Reynel & Rutaceae & $\mathrm{x}$ & & & & & & & & \\
\hline 130 & Zanthoxylum rigidum Humb. \& Bonpl. ex Willd. & Rutaceae & $x$ & $\mathrm{x}$ & $\mathrm{x}$ & $\mathrm{x}$ & $\mathrm{x}$ & & $x$ & $\mathrm{x}$ & \\
\hline 131 & Dyctioloma peruvianum Planch. & Sapindaceae & $\mathrm{x}$ & & & & & & & & \\
\hline 132 & Sapindus saponaria $\mathrm{L}$ & Sapindaceae & & & & & & & & & $x$ \\
\hline 133 & Serjania ampelopsis Pl. \& Lind. Ex Tr. \& Pl. & Sapindaceae & & & & & & & & & $x$ \\
\hline 134 & Serjania glabrata Kunth in H. B. K. & Sapindaceae & $\mathrm{x}$ & & & & & & & & \\
\hline 135 & Serjania grammatophora Radlk. aff. & Sapindaceae & & & & & & & & & $x$ \\
\hline 136 & Serjania inflata Poepp. \& Endl. & Sapindaceae & & & & & & & & & $x$ \\
\hline 137 & Sideroxylon obtusifolium (Roem. \& Schult.) T.D. Penn. & Sapotaceae & $\mathrm{x}$ & $\mathrm{x}$ & $x$ & $x$ & $\mathrm{x}$ & $x$ & $x$ & $\mathrm{x}$ & \\
\hline 138 & Solanum grandiflorum Ruiz \& Pav. & Solanaceae & & & & & & & & & $x$ \\
\hline 139 & Solanum riparium Persoon & Solanaceae & & & & & & & & & $x$ \\
\hline 140 & Taxa desconocido (JLMP-2269, 2280) & Solanaceae & & & & & & & & & $x$ \\
\hline 141 & Byttneria cordata Lam. . & Sterculiaceae & $\mathrm{x}$ & & & & & & & & \\
\hline 142 & Jacquinia mucronata Roem. \& Schult. & Theophrastaceae & $x$ & $\mathrm{x}$ & $x$ & $x$ & $x$ & $x$ & $x$ & $\mathrm{x}$ & \\
\hline 143 & Muntingia calabura $\mathrm{L}$. & Tiliaceae & & & & & & & $x$ & & \\
\hline 144 & Luehea paniculata Mart \& Zucc. & Tiliaceae & & & & & & & & & $x$ \\
\hline 145 & Celtis loxensisC. C. Berg & Ulmaceae & $x$ & & $x$ & & & & $x$ & & \\
\hline 146 & Guazuma ulmifolia Lam. & Ulmaceae & & $x$ & & & & & & & \\
\hline 147 & Trema micrantha (L.) Blume & Ulmaceae & & & & & & & & & $x$ \\
\hline 148 & Urera caracasana (Jacq.) Griseb. aff & Urticaceae & $x$ & & & & & & & & \\
\hline 149 & Aloysia scorodonioides (Kunth) Cham. & Verbenaceae & & $\mathrm{x}$ & & & & & & $x$ & \\
\hline 150 & Lantana sp. (JLMP-2066) & Verbenaceae & & & & & & & & & $x$ \\
\hline \multirow[t]{3}{*}{151} & Taxa desconocido (JLMP-2260) & & & & & & $\mathrm{x}$ & & & & \\
\hline & Total & & 47 & 42 & 46 & 35 & 39 & 29 & 40 & 50 & \\
\hline & Endémicas & & 10 & 12 & 15 & 14 & 10 & 8 & 11 & 14 & \\
\hline
\end{tabular}

